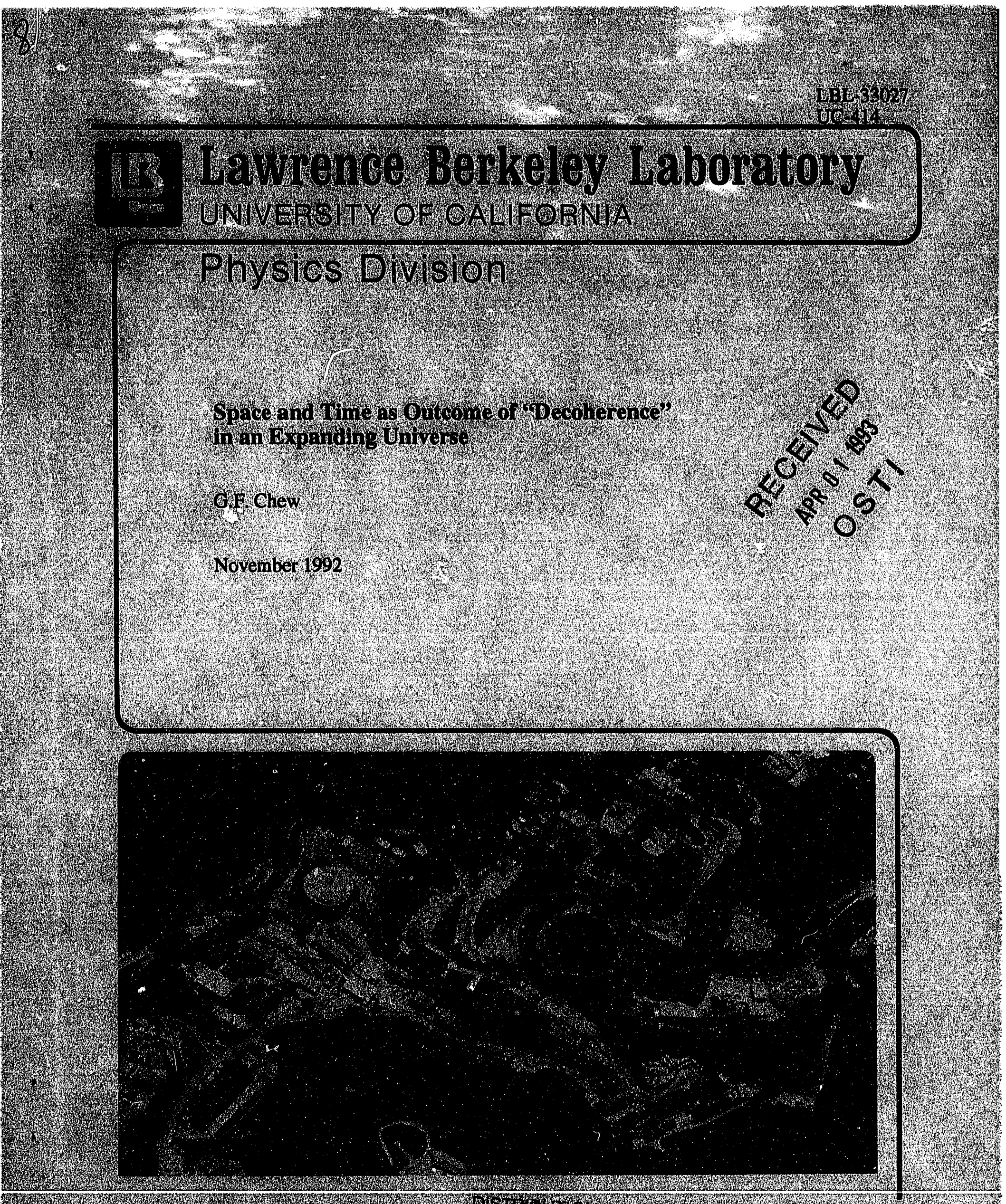

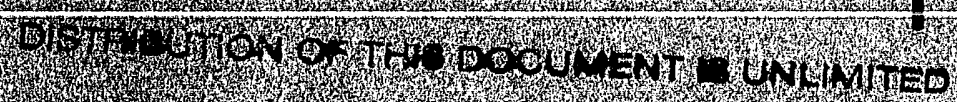
1.4. 


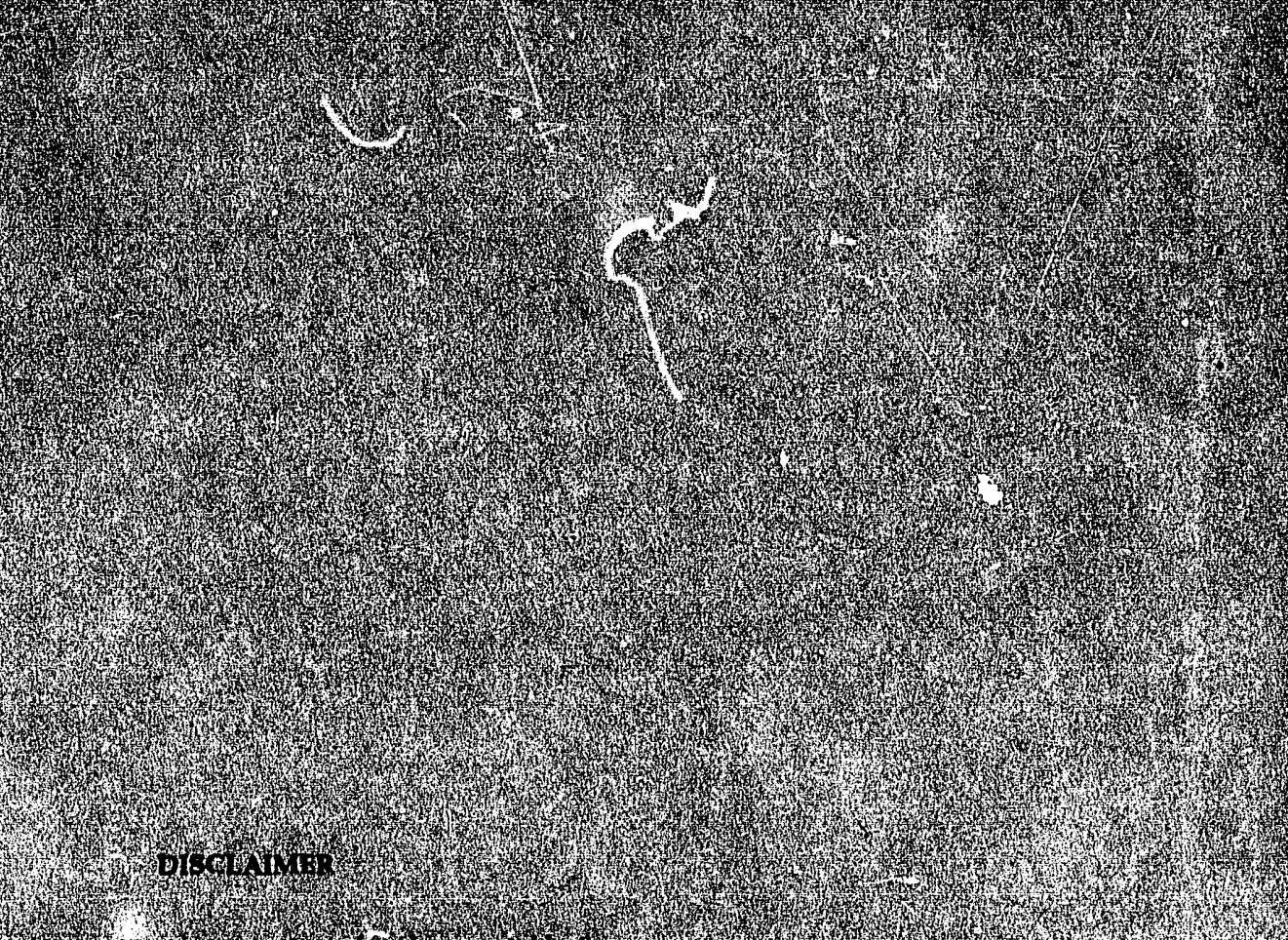

1.7.

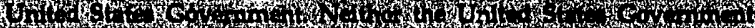

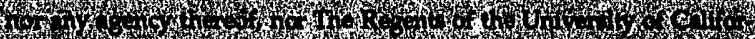

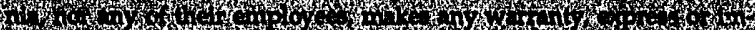

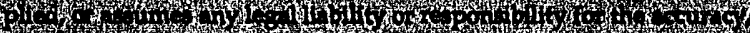
7.

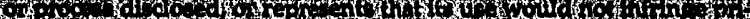

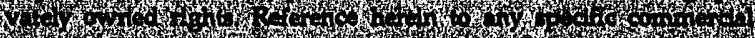

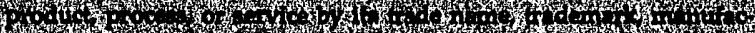

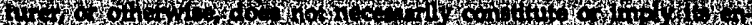
7.

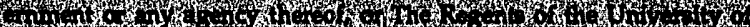

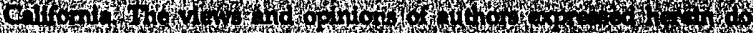

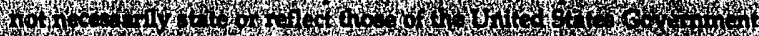

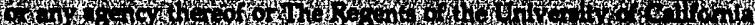
7. $+20$.

H17. thot

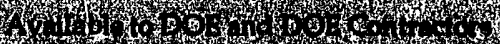

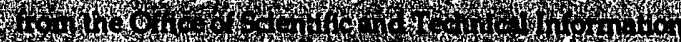
47.

7.

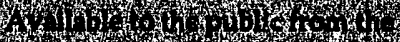

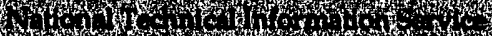

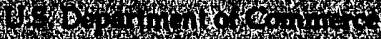

4.3.

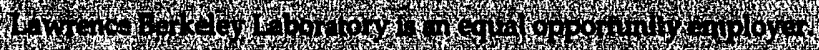


LBL-33027

UC-414

\title{
Space and Time as Outcome of "Decoherence" in an Expanding Universe
}

\author{
G.F. Chew
}

Physics Division

Lawrence Berkeley Laboratory

University of California

Berkeley, CA 94720

November 1992

\section{MASTER}

This work was supported by the Director, Office of Energy Research, Office of High Energy and Nuclear Physics, Division of High Energy Physics of the U.S. Department of Energy under Contract DE-AC03-76SF00098.

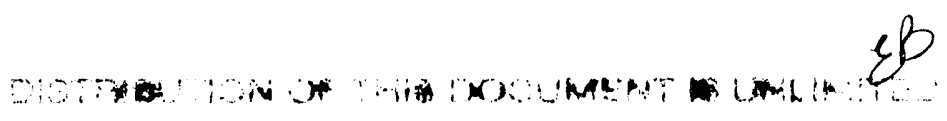


Despite the glorious achievements of 20th-century physics, obscurities persist. Each successive triumph has sharpened awareness of unresolved mysteries. Here are some recognized brain busters that motivate a quantum-cosmological model ${ }^{(1)}$ to be discussed today's talk:

a) Interpretation of quantum mechanics remains rooted in classical ideas about objective reality within space and time. Use of a less correct theory as underpinning for a more correct theory has spawned the puzzle of measurement. What, precisely, is meant by "measurement"? Is electromagnetism essential thereto? A growing collection of physicist-philosophers is expressing dissatisfaction with "collapse" of the quantum-mechanical state vector when "measurement" occurs.

b) "Objectivity", whatever this term means, minimally requires a "dilute" universe and thus, as stressed by Gell-Mann and Hartle, ${ }^{(2)}$ can be no more than a "relic of the big bang". At what stage did our expanding universe become suffciently dilute as to be approximately describable as built from separate objects? How accurate in the present universe is objectivity?

c) Ought physicists rest content with an a priori $3+1$ spacetime manifold, as the arena for objective reality? Is such an arena unaesthetically arbitrary? Does it create unnecessary mathematical difficulties for quantum theory?

d) Why is gravity so weak on the particle scale? $\left(G m_{p}^{2} \sim 10^{-38}\right.$ where $G$ is the gravitational constant and $m_{p}$ the proton mass, in units where $\hbar=c=1$ )

e) Does the notion of "before big bang" have meaning?

A complete description of the model to be discussed today is out of the question because of time limitation. Those who wish details may find them in preprints, LBL-32694 and 33081. I shall confine myself here to general characteristics.

The model avoids a priori classical ideas, even spacetime, by invoking a pair of established general principles that relate to each other:

A) Parameter largeness can endow physical approximations with accuracy.

B) Coherent states labeled by large parameters provide a quantum basis for classical ideas. ${ }^{(3)}$ 
Regarding Principle (A) one may recall how Newtonian mechanics, even though inexact, is successful for many purposes because of "largeness" of the inverse Planck constant and of the velocity of light. Objective reality within $3+1$ spacetime is an idea even more widely applicable than Newtonian mechanics. Our model attributes the extraordinary success of the objective view of nature (called "explicate order" by David Bohm) to huge but hitherto underappreciated parameters; these parameters (unequivocally finite) appear up front in the model.

As an example of Principle (B), (which you may find less familiar than (A) because coherent states are not usually discussed in standard quantum-mechanics courses), consider a Hilbert space capable of representing the rotation group $O(3)$ through 3 Hermitian operators $J_{1}, J_{2}, J_{3}$ as group generators. Generally speaking, the technical term "coherent state" refers to a special category of Hilbert-space vector that relates to some Lie group unitarily representable within the Hilbert space. In my $O(3)$ example, using a standard $\mid j, m>$ basis where $m$ is the eigenvalue of $J_{3}$ and $j(j+1)$ is the eigenvalue of $J_{1}^{2}+J_{2}^{2}+J_{3}^{2}$, the coherent state

$$
\left|\varphi, \theta>>_{j} \equiv e^{i J_{3} \varphi} e^{i J_{1} \theta}\right| j, j>
$$

represents, for $j \gg>1$, a classical rotator of angular momentum $j \hbar$ and axis direction $\theta, \varphi$ (polar coordinates). Largeness of $j$ is essential because fluctuation in angle of axis direction is $\sim 1 / j^{1 / 2}$; for $j \leqslant 1$ there is no classical interpretation of the coherent state (1). At large $j$, many operators become almost diagonal in angle - - almost "local" - - a phenomenon sometimes described as "decoherence". (Such terminology can be confusing in that a "coherent-state" basis here is essential to "decoherence".)

A second example of Principle (B) is the coherent photon state that represents a classical electromagnetic field. ${ }^{(4)}$ I shall not, for lack of time, write down this celebrated example explicitly but remark that the group in question is the so-called Heisenberg-Weyl group while the coherent-state parameters that must be large relate to the (mean) number of photons. It is typically the number of "soft" photons - - of frequency below discrete matter frequencies - - that grows so large as to generate "decoherence" of quantum electrodynamics into classical electro-magnetism.

In the Hilbert space of the quantum-cosmological model that I shall be discussing today, labels on basis vectors lack physical interpretation, but groups unitarily represented include not only the Heisenberg-Weyl group but the Poincaré group. Corresponding large-parameter coherent states are interpretable in terms of events and particles; a quantum-based meaning for classical objective reality in $3+1$ spacetime is thereby achievable. 
Dynamics is essential to generate requisite largeness and stability of parameters. In the model, a coherent-state representation is given of "big-bang" followed by "inflation" followed by a "particle era". Global time develops meaning after big bang while local relativistic spacetime, depending on particles, achieves significance after inflation. "Before" big bang there is absence of meaning for either space or time.

A key dynamically-changing parameter, labeling event-particle coherent states, corresponds to an expanding scale for the universe; in the present universe there is rough equivalence of this scale with the inverse Hubble parameter. Another key model parameter is constant, dimensionless and denoted by the symbol $\alpha$. The constant $\alpha$ is the model's only arbitrary parameter. The sign of $\alpha$ provides an "arrow of evolution" - - universe expansion rather than contraction. The magnitude of $\alpha$ turns out roughly to give the factor by which scale increased between big bang and the threshold of objectivity - - when the universe began to be representable as built from separate particles.

The model is characterized by zero total energy (defined as the expectation of Poincaré displacement generator). Assuming such to be achieved through balancing of negative gravitational energy against positive kinetic energy allows inference that $\alpha^{1 / 2}$ approximates the ratio between particle mass scale and big-bang scale. Needed for this inference is the standard guess for the latter as the Planck scale $\sim 10^{-33} \mathrm{~cm}$. It then follows that $\alpha \gtrsim 10^{34}$ - - huge although finite. Hugeness of $\alpha$ is seen to connect with weakness of gravity on the particle scale. More will be said later about gravity within the model.

It is hugeness of the arbitrary parameter $\alpha$ that underpins objective reality and spacetime in our model. This hugeness, of course, is a symptom of model incompleteness; a better model would determine $\alpha$. (I remark that a Cambridge-originating ${ }^{(5)}$ crew of combinatoriallyoriented investigators have for many years been asserting as relevant to physics the prime number $2^{127}-1 \sim 10^{38}$. Combinatorics can generate huge numbers.)

An LBL investigation, in collaboration with Henry Stapp and two students, Paul Masson and Leehwa Yeh, and extending over much of the past decade, has revealed a surprisinglysimple Hilbert space to be capable both of housing the requisite coherent states and of admitting appropriate evolutionary dynamics: The model invokes a product of 16 simple Fock spaces. Fock-space basis vectors are labeled by positive integers ("occupation numbers"), are sequentially connected by annihilation-creation operators and relate naturally to graphs. (You no doubt have encountered Fock space in description of a harmonic oscillator; in fact this Hilbert space is more widely useful). All operators on the space are representable through annihilation-creation, and in our model each annihilation or creation operator is a 4 $\times 4$ matrix, allowing topological connection with graphs embedded in oriented 2-dimensional 
surfaces. ${ }^{(6)}$

Our attention was initially directed to this system by the dual topological models that led to string theory. Later Eyvind Wichmann made us appreciative of the algebraic connection between $4 \times 4$ matrices and complex conformal transformations in $3+1$ spacetime. The real conformal group encompasses the Poincaré group and further includes scale change. Ever since Wichmann's intervention, our emphasis has been on coherent states related to the complex conformal group. For a long period we were baffled by the model doubling of real conformal generators but now recognize this doubling as providing simultaneous representation of spacetime in the "large" sense of cosmology and spacetime in the "small" sense of particles. I shall return to the coexistence in our model of two complementary spacetimes.

At the risk of belaboring the point, I emphasize absence of a priori physical significance for the 16 valued label on our Fock space; our physics emerges from large coherent-state parameters that exhibit dynamical stability. I also caution against inappropriate invocation of an a priori notion of "number of dynamical degrees of freedom". A product of 16 simple Fock spaces might be thought to correspond to a system with 16 degrees of freedom - - like 16 harmonic oscillators. It then sounds futile for the model to attempt representation of the entire universe. But a Fock space is immensely larger than the corresponding (classical) phase space and this size is exploited through hugeness of the constant $\alpha$ and a dynamics that generates an expanding scale. Our dynamics does not correspond to that of a set of oscillators.

Model capacity to represent evolution associates with a parameter akin to but different from time. Evolution is described through a parameter $\tau$, that spans the real line, together with a Schrödinger-type equation of motion:

$$
\frac{\partial}{\partial \tau}|\tau>=-i \mathcal{H}| \tau>
$$

The Hermitian operator $\mathcal{H}$ is called the "evolution generator", with the state vector $|\tau\rangle$ describing the entire universe at $\tau$. After extensive search a promising evolution generator has been found: a highly symmetric quartic in annihilation-creation operators. This is an operator (with continuous eigenvalues) that is very different from a harmonic-oscillator Hamiltonian.

Fifteen bilinear operators commute with $\mathcal{H}$, corresponding to symmetry under homogeneous complex conformal transformations that include complex Lorentz transformations and dilations (change of scale). All symmetry generators except that for dilation are assigned zero expectation values. The parameter $\alpha$ is defined to be the expectation of the dilation 
generator; the sign of $\alpha$ controls the "arrow of evolution". A boundary condition on $\mid \tau>$ in the limit $\tau \rightarrow-\infty$ corresponds to a universe that has "always been expanding" (even before big bang, although I shall explain later that "scale" is definable only after big bang.)

I remark that the evolution parameter $\tau$, unlike time, is unaffected by either Lorentz transformation or dilation. Meaning for time in our model is not a priori but achieved through dynamics via coherent states.

A condensed notation for the model's coherent states, based on a group whose generators combine complex Heisenberg-Weyl with compact homogeneous complex-conformal generators, is

$$
\mid s, \widehat{n}_{e} R(\tau)>_{\alpha}
$$

These Hilbert-space vectors are called "event-particle coherent states." Here $R(\tau)$ is the scale parameter while $\hat{n}_{e}$ is a complex 4-vector of unit norm that locates an event within a pair of coexisting $3+1$ spacetimes. Complex extension of Poincaré and dilation groups has led to a noncompact absolute spacetime of scale $R(\tau)$ together with a compact relativistic spacetime of radius $R(\tau)$. The absolute time of an event is

$$
t_{e}=R(\tau) \cosh \beta_{e}
$$

where $\vec{\beta}_{e}$ is a Lorentz boost parameter spatially locating the event at

$$
\vec{r}_{e}=R(\tau) \frac{\vec{\beta}_{e}}{\beta_{e}} \sinh \beta_{e} .
$$

The index $s$ designates a set of particles, some of which were created in Event $e$ - - the "last" event occurring in creation of the particle set $s$. Particles in this set are not directly located in noncompact absclute space but have absolute velocities (as well as masses) and locate in the compact spacetime relative to Event $e$ and to each other.

The theory is invariant with respect to shift of compact-spacetime origin (that is what "relativistic" means) but "big-bang" is an event unaccompanied by particles ( $s$ for big bang is the null set) that locates at the origin $\left(\vec{\beta}_{e}=0\right)$ of absolute (noncompact) space. By choice of $\tau$ origin big bang occurs at $\tau=0$.

During an evolution interval immediately subsequent to big bang,

$$
0<\tau \lesssim \ln \alpha
$$

the universal state vector is approximatable as the outcome of big bang followed merely by exponential expansion of scale,

$$
R(\tau)=R(0) e^{\tau}
$$


through a factor of order $\alpha$. Following standard terminology, I refer to this dynamicallysimple evolution interval following big bang as "inflation." (7) Note that the absolute time of big bang is not zero but $R(0)$, which we are guessing to be of the order of the Planck time $\sim 10^{-43} \mathrm{sec}$. There is no meaning for time before $\tau=0$ because here the universal state vector cannot be approximated through event-particle coherent states. (Strictly speaking, time does not become meaningful until scale substantially exceeds $R(0)$.)

For $\tau \gtrsim \ln \alpha$ the single-parameter approximation breaks down and one requires multiparameter coherent states representing particles generated in events that spread through absolute space. The universal state vector may be written

$$
\left|\tau>=\sum_{s} \int d \hat{n}_{e} \psi_{s}\left(\widehat{n}_{e}, \tau\right)\right| s, \widehat{n}_{e} R(\tau)>_{\alpha}
$$

Roughly speaking, the particle era begins when $R(\tau)$ exceeds $\alpha R(0)$; the model accommodates a "universe built of particles" when scale becomes large compared to this transition scale. The transition scale is presumed to correspond to a particle density such that mean interparticle separation is comparable to particle Compton wavelength and kinetic energy density is of order $m^{4}$, where $m$ is particle mass. (At higher density individual particles lose meaning because widths become larger than masses.) Here I am assuming a balancing of negative gravitational energy against positive kinetic energy so that density is "critical" and diminishing in proportion to $R^{-2}(r)$ as scale increases. As noted earlier, one then infers

$$
\alpha \sim \frac{1}{G m^{2}} \gtrsim 10^{34}
$$

assuming particle mass to be less than or of the order of $100 \mathrm{GeV}$.

The particle concept represents stability (an electron is an electron is an electron) while the event concept represents change (as when a neutrc: decays into proton, electron, neutrino). There is tension between these concepts: Particles are born and die in events (any collision is an event); finite duration of particle existence means Heisenberg uncertainty in mass and consequent blurring of identity, as noted in my interpretation of the transition scale $\alpha R(0)$. Event-particle coherent states associate the two conflicting notions with canonicallyconjugate noncommuting operators so that focus on one notion blurs the other. Hugeness of scale in the present universe, where $R(\tau)$ is of order $10^{60} R(0)$ and thus far beyond the model's transition scale, allows event and particle concepts to be simultaneously (approximately) viable and renders our universe objectively observable. Prior to and during inflation there were no particles and no objective reality. According to our model, space was without meaning. 
Figure 1 summarizes the foregoing by exhibiting logarithmic intervals of the scale parameter.

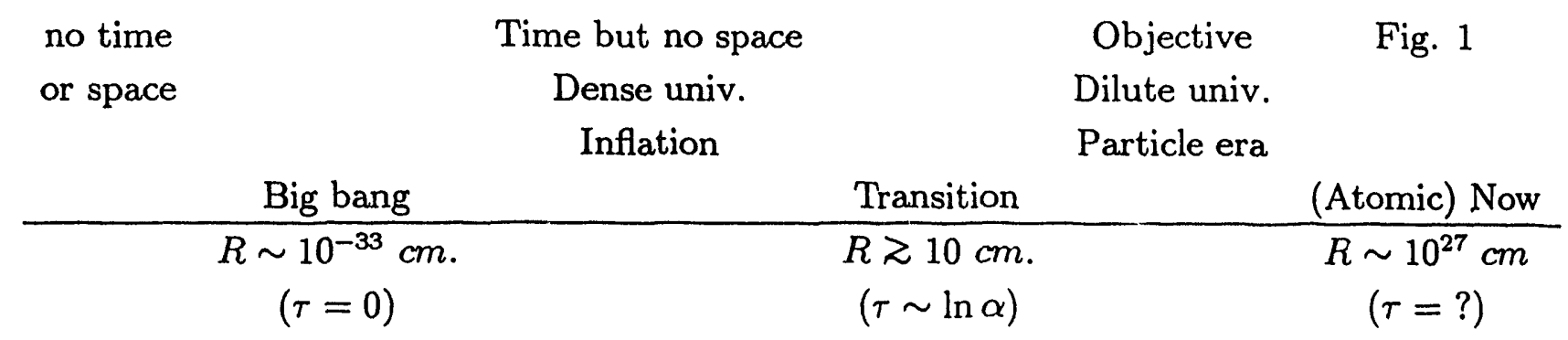

Standard cosmology suggests that, once a large number of particles has developed (following the inflation interval), most $\tau$ dependence of the universal state vector associates with creation-annihilation of particles and relatively little with scale expansion. Scale is expected to continue growing but at a rate much slower than exponential. The value of $\tau$ for the present universe, although not yet known, is correspondingly expected to be huge.

An essential feature of event-particle coherent states is their phase, which depends on compact-spacetime locations of particles relative to event location. The phase plays the role of action and, at huge scale, stationary phase yields classical "straightline" compactspacetime particle trajectories that originate in events. Such relativistic trajectories are simple in a special Lorentz frame centered on an event but lack meaning for the absolute noncompact spacetime that houses all events (each with its own special frame). If meaning can be found for "absolute" trajectory of a particle, there must be reference to events involving other particles; influence on the absolute trajectory from spatial location and energy-momentum of other particles is then to be expected. The problem is not yet solved, and I am hopeful (as an incorrigible optimist) that absolute-spacetime curved trajectories will be found compatible with gravitation. The first quantitative test of the model promises to be its capacity to describe gravity.

Further down the road will be calculation of particle masses, spins, etc. Such computations promise to be difficult. The zero-mass, spin-1 photon might prove an exception; event-particle coherent states "on the light cone" are already being studied. The mystery of "measurement" and "consciousness" interlocks with the mystery of "soft photons" engaged in "gentle events" during the "atomic era" of the universe, ${ }^{(8)}$ which logarithmically-speaking began rather recently ( $\sim 5$ orders of scale magnitude ago). 
I close with a schematic approximate decomposition of the evolution generator,

$$
\mathcal{H} \underset{R(\tau) \gg>\alpha R(0)}{\underset{\Delta u g e}{\approx} \alpha} \sum_{\Delta(e, \tau)} \int d \widehat{n}_{e} \mathcal{H}_{\Delta(\widetilde{e}, \tau)}^{R(\tau)}
$$

that compactly (although without rigor) conveys the essence of the model's explanation" of objectivity. I first encountered this type of formula in a celebrated 1962 paper by Haag ${ }^{(9)}$ that elucidated the BCS theory of superconductivity. Because of hugeness of a parameter, the Hilbert space in a coherent-state basis selected by dynamics ${ }^{(3)}$ effectively "shrinks" (approximately) to a direct product of smaller spaces that are distinguished by classical labels. This shrinkage is sometimes called "decoherence". Subspaces with labels that differ by more than a small amount are almost disconnected - - not coupled by $\mathcal{H}$. In our model $\widehat{n}_{e}$ distinguishes different regions in spacetime, each surrounding some event, and $\Delta(\widehat{e}, \tau)$ denotes corresponding subsets of particles. Formula (9) then states that, in a huge-scale universe, evolution proceeds approximately on a local basis - - each spacetime region evolving (approximately) independently of distant regions. Herein lies underpinning for an objective viewpoint based on local spacetime.

Gravity constitutes a correction to objectivity, but gravity is weak on the particle scale. In our model this weakness correlates via the constant $\alpha$ with hugeness of cosmological scale throughout the particle era. Two mysteries reduce to one: Hugeness of $\alpha$.

\section{References}

1. G.F. Chew, Lawrence Berkeley Laboratory preprints LBL-32694 and 33081, Berkeley (1992).

2. M. Gell-Mann and J. Hartle, Proceedings of the 3rd International Symposium on the Foundations of Quantum Mechanics in the Light of New Technology, ed. by S. Kobayashi, H. Egawa, Y. Murayama and S. Nomura, Physical Society of Japan, Tokyo (1990).

3. L. G. Yaffe, Rev. Mod. Phys. 54, 407 (1982).

4. R. Glauber, Phys. Rev. Lett. 10, 84 (1963).

5. E.W. Bastin and C.W. Kilmister, Proc. Camb. Phil. Soc. 50, 278 (1954) and 51, 454 (1955). E.W. Bastin (edit) "Quantum Theory and Beyond", Cambridge 1971, p.213

6. G.F. Chew and V. Poénaru, Phys. Rev. D32, 2783 (1985). 
7. A. Guth, Phys. Rev. D23, 347 (1981).

8. G.F. Chew, Phys. Rev. A45, 4312 (1992).

9. R. Haag, Nuovo Cimento XXV, 287 (1962). 


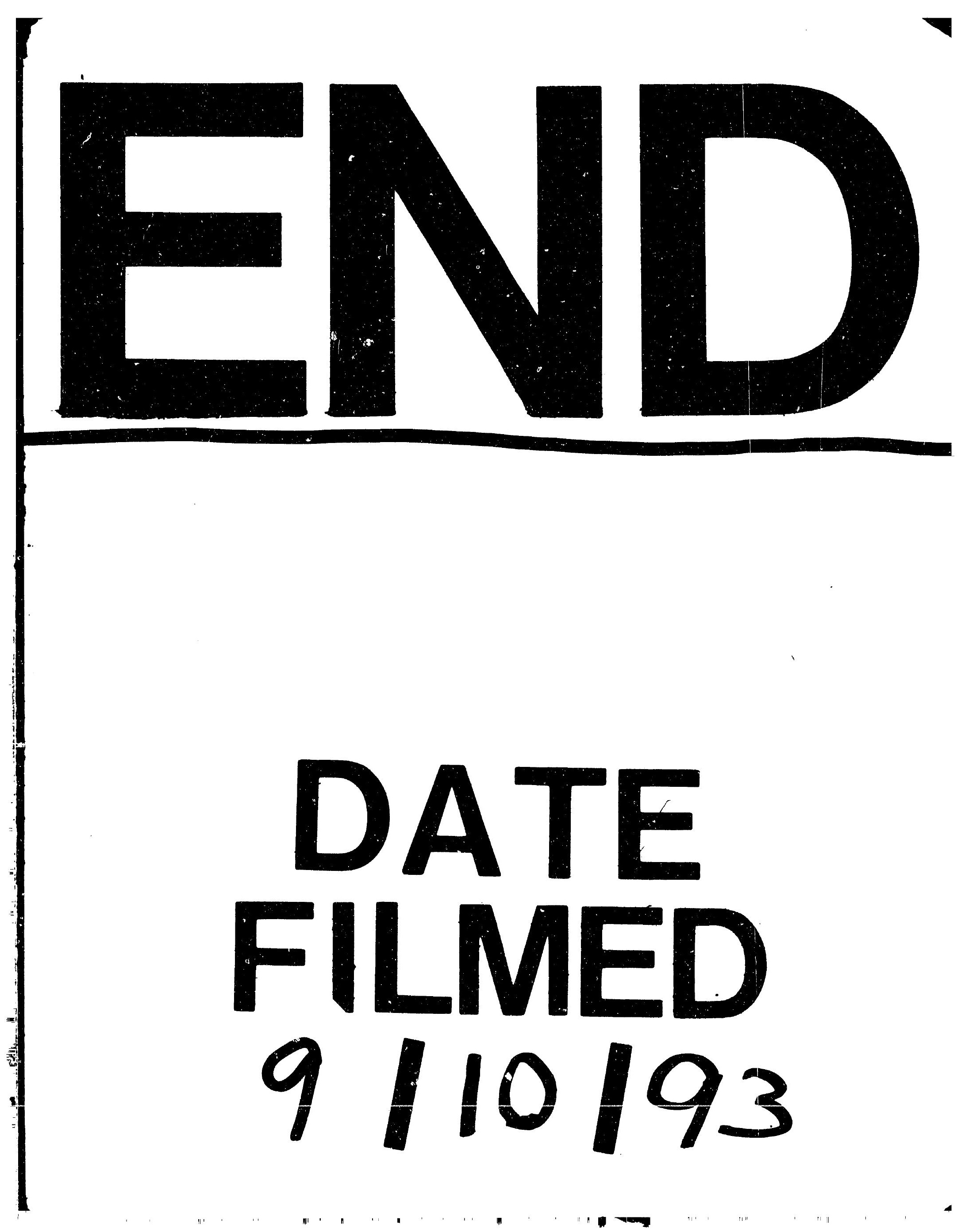



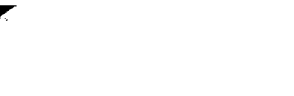

(
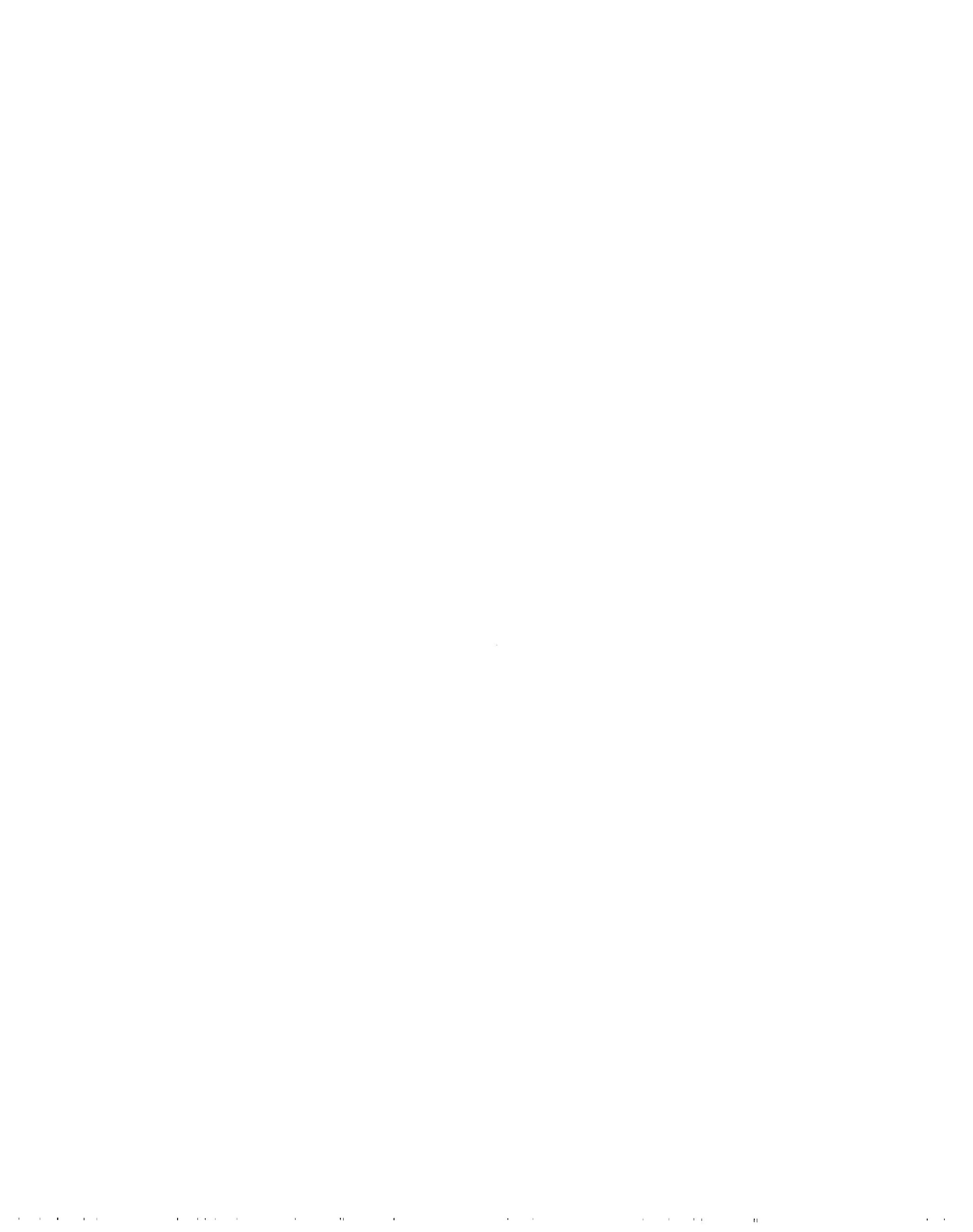